Int. J. Electrochem. Sci., 15 (2020) $6269-6289$

\title{
Au@Pt Hybrid Nanorods Encapsulated in B, S dual-doped Graphene as Highly Sensitive Immunosensing Platform for Electrochemical Determination of Aflatoxin $\mathbf{B}_{1}$
}

\author{
Xianlan Chen ${ }^{1,2}$, Huajian $\mathrm{Li}^{3}$, Guowei Zhang ${ }^{1,2}$, Shaoping Feng ${ }^{1,2, *}$, Guangming Yang ${ }^{1,2, *}$, Ling Shi ${ }^{1,2}$, \\ Wei Liu ${ }^{1,2, *}$, Guiyang, Liu $^{1,2}$ and Haibo Pan ${ }^{4}$ \\ ${ }^{1}$ School of Science, Honghe University, Mengzi, 661199, Yunnan, China; \\ ${ }^{2}$ Key Laboratory of Natural Pharamaceutical \& Chemical Biology of Yunnan Province, Mengzi, \\ 661199, Yunnan, China \\ ${ }^{3}$ College of Food Science and Technology, Yunnan Agricultural University, Kunming, China \\ ${ }^{4}$ Fujian Key Lab of Medical Instrument \& Pharmaceutical Technology, Yishan Campus, Fuzhou \\ University, Fuzhou, Fujian, 350002, China \\ *E-mail: shaopingfeng@126.com; 13489086418@163.com; yangguangmingbs@126.com; \\ liuwei4728@126.com
}

doi: $10.20964 / 2020.07 .19$

Received: 13 February 2020 / Accepted: 9 April 2020 / Published: 10 June 2020

\begin{abstract}
A specific electrochemical immunosensor for ultra-sensitive determination of Aflatoxin $\mathrm{B}_{1}\left(\mathrm{AFB}_{1}\right)$ was developed according to a dual enhancing strategy. The dual-doped graphene (BS-G) was obtianed by a simple microwave-assisted hydrothermal approach, sulfur and boron affinity for peanut-shaped Au@Pt NRs embedded into BS-G nanosheets, ultrathin BS-G effectively enwrapped most of the individual $\mathrm{Au} @$ Pt NRs with excellent dispersibility. It is noteworthy that two faces at the interface between (002) crystal faces of BS-G and (111) planes of Au@Pt NRs are compatible, accelerating the charge transfer between the hybrid nanocatalysts and $\mathrm{AFB}_{1}$. Moreover, the highly dispersed $\mathrm{COOH}$ groups and defect sites of the BS-G precisely control the orientation of anti-AFB $\mathrm{B}_{1}$ immobilized on the sensor surface, meanwhile, the strong electronic interactions endow the $\mathrm{Au} @ \mathrm{Pt}$ NRs/BS-G hybrid nanocatalysts with remarkable biocompatibility and multivalent affinity interactions with the high molar ratio of anti$\mathrm{AFB}_{1}$ for enhanced immunoreactions. Thus, the designed immunosensor exhibits ultra-efficient electron-transfer abilities, excellent sensitivity, and favorable selectivity, the detection limit is 0.0052 $\mathrm{ng} \cdot \mathrm{mL}^{-1}$ and the linear range is $0.025-60.00 \mathrm{ng} \cdot \mathrm{mL}^{-1}$.
\end{abstract}

Keywords: AFB $;$; B, S dual-doped grapheme; Au@Pt NRs; Au@Pt NRs/BS-G hybrid nanocatalysts, Electrochemical immunosensor

FULL TEXT 
(C) 2020 The Authors. Published by ESG (www.electrochemsci.org). This article is an open access article distributed under the terms and conditions of the Creative Commons Attribution license (http://creativecommons.org/licenses/by/4.0/). 